\title{
Conduction band offsets in CdZnSSe/ZnSSe single quantum wells measured by deep level transient spectroscopy
}

\author{
P. F. Baude, M. A. Haase, G. M. Haugen, K. K. Law, T. J. Miller, and K. Smekalin \\ $3 M$ Company, 3M Center 201-1N-35, St. Paul, Minnesota 55144 \\ J. Phillips and P. Bhattacharya ${ }^{a)}$ \\ Solid State Electronics Laboratory, Department of Electrical Engineering and Computer Science, \\ The University of Michigan, Ann Arbor, Michigan 48109-2122
}

(Received 4 March 1996; accepted for publication 12 April 1996)

Conduction-band offsets in wide-band-gap CdZnSSe/ZnSSe single quantum well structures have been characterized by deep level transient spectroscopy (DLTS) measurements. $50 \AA$ thick $\mathrm{Cd}_{0.3} \mathrm{Zn}_{0.7} \mathrm{~S}_{0.06} \mathrm{Se}_{0.94}$ single quantum wells with $\mathrm{ZnS}_{0.06} \mathrm{Se}_{0.94}$ barriers were grown by molecular beam epitaxy on GaAs substrates. A thermal emission energy from the quaternary wells of $179 \pm 10$ $\mathrm{meV}$ was measured. This corresponds to a conduction-band offset energy of $\sim 251 \pm 20 \mathrm{meV}$. (C) 1996 American Institute of Physics. [S0003-6951(96)02525-9]

Short wavelength semiconductor lasers have attracted much attention since the first demonstration of ZnSe based injection lasers by Haase et al. ${ }^{1}$ in 1991. Rapid progress in the area of II-VI wide-band-gap material growth has resulted in room-temperature continuous-wave (cw) operation with lifetimes in excess of $3 \mathrm{~h}^{2}$ These devices typically operate at about $510-530 \mathrm{~nm}$ and consist of $\mathrm{Cd}_{x} \mathrm{Zn}_{1-x}$ Se quantum wells with a $\mathrm{Zn}_{1-x} \mathrm{~S}_{x} \mathrm{Se}$ and $\mathrm{Mg}_{1-x} \mathrm{Zn}_{x} \mathrm{~S}_{y} \mathrm{Se}_{1-y}$ guiding and cladding layer, respectively. ${ }^{3-5} \mathrm{~A}$ wide range of lasing wavelengths $(460-550 \mathrm{~nm})$ can be realized in this system by varying the cadmium concentration from $0 \%, \mathrm{ZnSe}$, to more than $40 \%$.

Design of II-VI ZnSe based laser devices requires knowledge of the conduction- and valence-band offsets. Several heterointerfaces are present in the most common wideband-gap ZnSe based laser devices, including the III-V (GaAs substrate) $-\mathrm{ZnSe} n$-type interface, the quantum well double heterostructure and the $p$-type $\mathrm{ZnSe}-\mathrm{ZnTe}$ contact. Numerous experimental and theoretical band offset studies have been reported on a wide variety of II-VI based heterojunctions ${ }^{6,7}$ and quantum wells. ${ }^{8-12}$ However, to the best of our knowledge there is no definitive experimental data on the conduction- and valence-band offsets occurring at the quantum well/guiding layer interface. In this letter, we report experimental results of conduction-band offsets in pseudomorphic $\mathrm{Cd}_{0.3} \mathrm{Zn}_{0.7} \mathrm{~S}_{0.06} \mathrm{Se}_{0.94} / \mathrm{ZnS}_{0.06} \mathrm{Se}_{0.94} \quad$ single quantum well structures measured by deep level transient spectroscopy (DLTS).

The basis for this technique, of measuring band offsets with DLTS, is that the potential variation of a quantum well is similar to that of a deep level, with some obvious differences. ${ }^{13,14}$ From a detailed balance between thermal capture and emission rates of electrons from a quantum well, the emission rate of electrons is given by ${ }^{14}$

$$
e_{n}=\left(16 \pi^{3 / 2} / 3 h^{3}\right) m_{e}^{*} \chi(k T)^{1 / 2}(\Delta E)^{3 / 2} \exp \left(-\Delta E_{e} / k T\right),
$$

where $\Delta E_{e}$ is the electron emission energy for the conduction band, $m_{e}^{*}$ is the effective mass in the well material, and

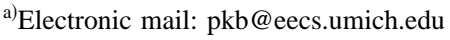

$\chi$ is a parameter related to the capture of carriers by the wells. ${ }^{13}$ The thermal emission energy of carriers from a quantum well is related to the band offset.

We now consider a single quantum well in the depletion region of a Schottky barrier. The width of the Schottky barrier depletion region that contains the quantum well is given by

$$
W^{2}=W_{0}^{2}\left(1+2 n_{w} L L_{w} / N_{D} W_{0}^{2}\right)
$$

where $W_{0}^{2}=\left(2 \varepsilon / q N_{D}\right) V$ is the depletion region width of in the absence the well, $L_{w}$ is the thickness of the well, $L$ is the thickness of the barrier from the Schottky contact to the well, $N_{D}$ is the net donor density in the barrier, and $V$ is the sum of the applied reverse bias and the built-in voltage drop, $V_{b i}$. The DLTS signal, $s(t)$, for rate windows $t_{1}$ and $t_{2}$ is given by $^{15}$

$$
s(t)=C_{0} \frac{n_{w}}{N_{D}} \frac{L L_{w}}{W_{0}^{2}}\left[\exp \left(-e_{n} t_{1}\right)-\exp \left(-e_{n} t_{2}\right)\right],
$$

where the capacitance $C_{0}$ corresponds to the depletion width $W_{0}$.

The structure used in this study was grown by molecular beam epitaxy on a (100) silicon doped GaAs substrate. The growth procedure has been described in detail previously. ${ }^{16,17}$ The cross section of the structure is illustrated in the inset of Fig. 1. A $3000 \AA$ thick $\mathrm{ZnS}_{0.06} \mathrm{Se}_{0.94}$ chlorine doped barrier layer was first grown on the $n$-type GaAs substrate followed by the growth of a compressively strained single $\mathrm{Cd}_{0.3} \mathrm{Zn}_{0.7} \mathrm{~S}_{0.06} \mathrm{Se}_{0.94}$ quantum well. A second $n$-type $\mathrm{ZnS}_{0.06} \mathrm{Se}_{0.94}(1000 \AA)$ was then grown as the top barrier. The CdZnSSe quantum well thickness was determined, by analyzing cross-sectional high resolution transmission electron microscopy images, to be about $50 \AA$ A. The band gap of the $\mathrm{ZnS}_{0.06} \mathrm{Se}_{0.94}$ barrier was evaluated from optical reflectance measurements to be $2.70 \mathrm{eV}$, corresponding to a sulfur concentration of $6 \%$. The CdZnSSe quantum well $\mathrm{ZnSSe}$ barrier interface is essentially identical to the quantum well/guiding layer interface of the most common II-VI based blue-green laser diodes operating at $515 \mathrm{~nm}$.

Room-temperature photoluminescence performed on the structure using the $457 \mathrm{~nm}$ line of an argon-ion laser re- 


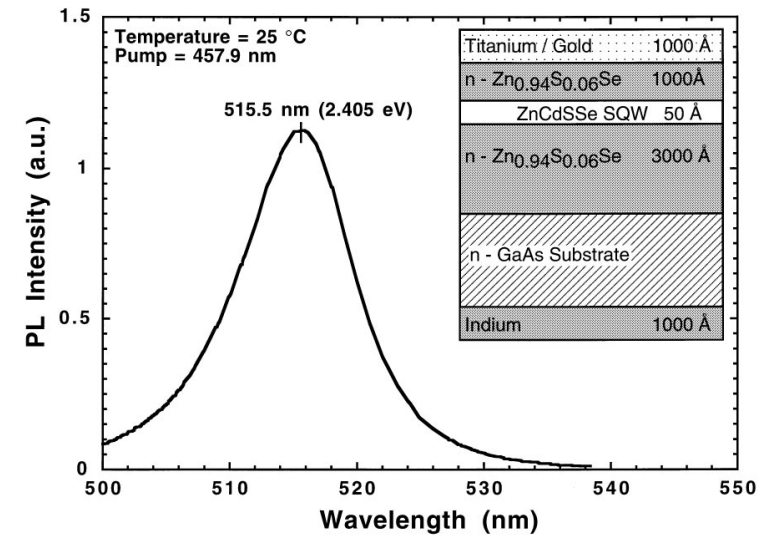

FIG. 1. Photoluminescence spectra of $\mathrm{ZnSSe} / \mathrm{CdZnSSe}$ single quantum well. The inset shows the diode structure, with the SQW, for DLTS measurements.

vealed a strong luminescence peak at $515.4 \mathrm{~nm}(2.406 \mathrm{eV})$, shown in Fig. 1, which is attributed to the CdZnSe single quantum well. This emission is the result of electron-hole recombination between the first electron and heavy-hole subbands. The energy difference between the barrier layer and the effective quantum well band gap is then $294 \mathrm{meV}$.

Schottky diodes were prepared by evaporating titanium/ gold (1000/2000 ̊, respectively) electrodes on the $n$-type $\mathrm{ZnS}_{0.06} \mathrm{Se}_{0.94}$ top layer. The backside GaAs substrate was metallized with $1000 \AA$ of palladium. The capacitance was measured as a function of applied reverse bias to estimate the effective doping concentration and to determine the approximate depth of the well. As illustrated in Fig. 2 the quantum well is located about $1000 \AA$ (inset) beneath the surface as expected from previously determined growth rates. The carrier concentration, $N_{D}-N_{A}$, in the barrier layer is about 3 $\times 10^{17} \mathrm{~cm}^{-3}$.

It is important to ensure that the observed DLTS signal represents electron emission from the quantum well and not deep level defects in the barrier material. There have been numerous reports of deep level defect identification in both $n$ - and $p$-type ZnSe based thin films. ${ }^{18-23}$ In chlorine doped $\mathrm{ZnSe}$ Karczewski et al. ${ }^{22}$ report two defect energy levels,

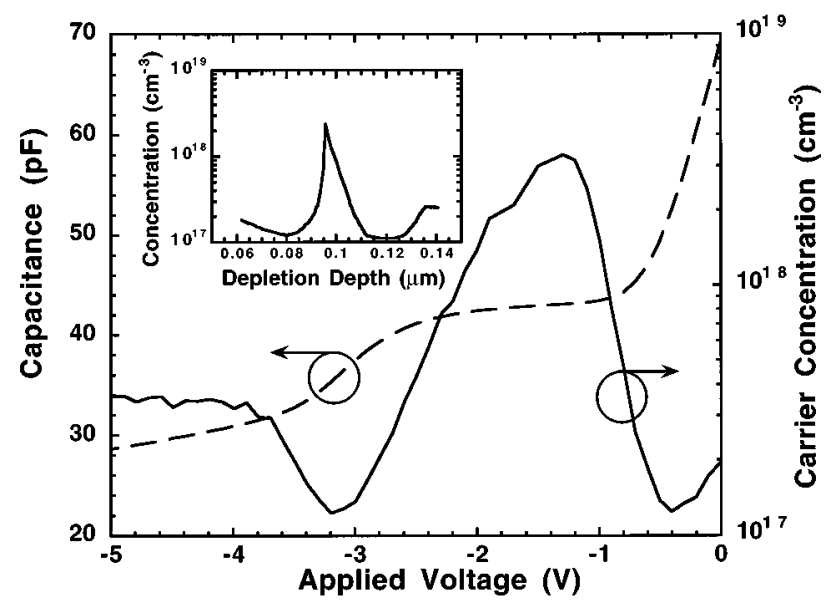

FIG. 2. Measured variation of capacitance and carrier concentration of the SQW diode with applied bias. The inset shows the variation of carrier concentration with depletion width.

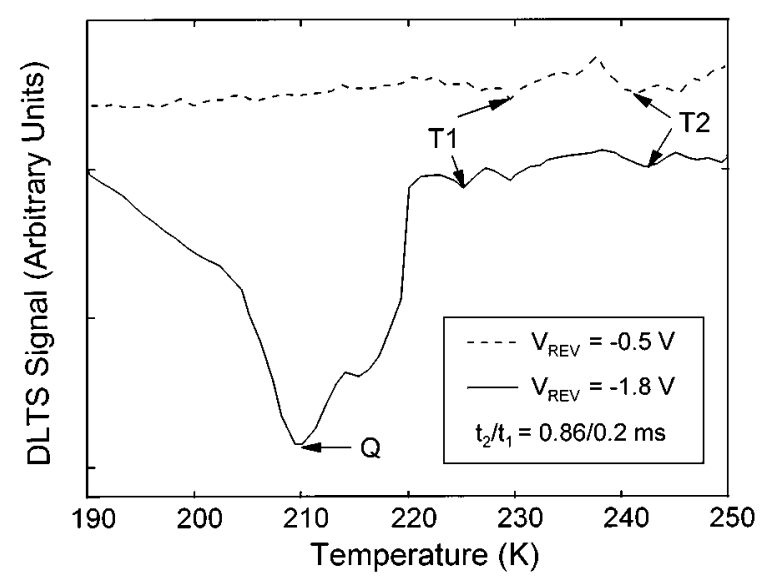

(a)

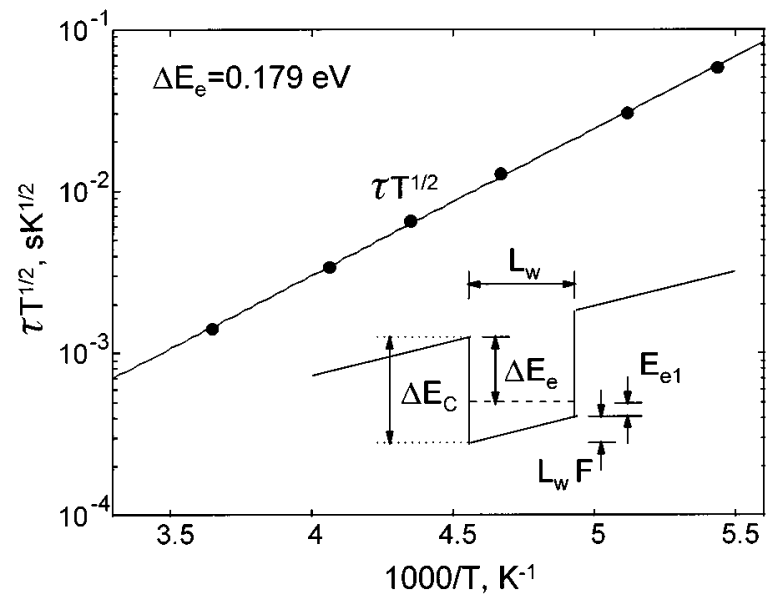

(b)

FIG. 3. (a) DLTS data for two quiescent reverse biases; (b) Arrhenius plot of electron emission rate vs inverse temperature. The slope of the plot gives the emission energy, $\Delta E_{e}$, indicated in the inset with other relevant parameters.

300 and $510 \mathrm{meV}$ below the conduction band edge. Similar values have been observed in undoped $\mathrm{ZnSe}$ grown by molecular beam epitaxy. ${ }^{19}$ The dominant emission signal in our DLTS measurements did not correspond to any previously reported deep level defect energy in II-VI materials.

Typical DLTS data at specified bias and rate windows, obtained from our samples, are shown in Fig. 3(a). The figure shows two scans at different reverse biases, so that the quantum well is included or excluded in the depletion region. The biases are chosen from the measured capacitancevoltage data of Fig. 2. The peaks labeled $T_{1}$ and $T_{2}$ are likely to be from electron traps, though they do not correspond to the 0.3 and $0.51 \mathrm{eV}$ levels seen by other authors. ${ }^{19,22}$ The peak labeled Q is conspicuously absent in the scan excluding the quantum well. Similar features are seen for other rate windows. We therefore conclude that the peak $\mathrm{Q}$ results from electron emission from the $50 \AA \mathrm{Cd}_{0.3} \mathrm{Zn}_{0.7} \mathrm{~S}_{0.06} \mathrm{Se}_{0.94}$ quantum well. Since our primary objective in this study was to determine the CdZnSSe/ZnSSe heterojunction band offset, we did not investigate the bulk traps labeled $T_{1}$ and $T_{2}$ further. The Arrhenius plot for the quantum well emissions obtained with different rate windows is shown in Fig. 3(b). An activation energy of $\Delta E_{e}=179 \pm 10 \mathrm{meV}$ is obtained from 
the slope of the plot. The thermal emission energy $\Delta E_{e}$ is related to the conduction band offset by the approximate relation

$$
\Delta E_{c}=\Delta E_{e}+E_{e 1}+L_{w} F,
$$

where the parameters on the right-hand side are depicted in the inset of Fig. 3(b). F is the electric field across the well region due to the applied reverse bias. There are obvious sources of error in this simple formulation, the principle ones being the spread in the subband energies and the excess energy of carriers above the barrier during emission, or reduced energy due to tunneling.

The value of $E_{e 1}$ is obtained from theoretical analysis including the effect of biaxial strain (misfit $=0.02436$ ). The value of $F$ is obtained from the applied reverse bias of the DLTS sweeps and the depletion region width calculated from (2). The value of $\Delta E_{c}$ derived from Eq. (4) is $269 \pm 20$ meV using $\Delta E_{e}=179 \pm 10 \mathrm{meV}, \Delta E_{e 1}=51 \pm 5 \mathrm{meV}, L_{w}$ $=50 \AA$, and $F=7.7 \times 10^{4} \mathrm{~V} / \mathrm{cm}$. With $\Delta E_{g}=2.70-2.34$ $=0.36 \mathrm{eV}$, we get $\Delta E_{c} / \Delta E_{g}=0.75 \pm 0.05$. Consequently we get $\Delta E_{v} / \Delta E_{g}=0.25 \pm 0.05$ and $\Delta E_{v}=91 \pm 20 \mathrm{meV}$ for the pseudomorphic $\mathrm{Cd}_{0.3} \mathrm{Zn}_{0.7} \mathrm{~S}_{0.06} \mathrm{Se}_{0.94} / \mathrm{ZnS}_{0.06} \mathrm{Se}_{0.94}$ heterostructure. The experimental results obtained in this work are in close agreement with theoretical predictions of band offsets in the $\mathrm{CdZnSe} / \mathrm{ZnSSe}$ strained-layer system reported by Wu et al. ${ }^{11} \mathrm{Wu}$ has calculated a thermal emission energy of about $140 \mathrm{meV}$ from the first electron subband in a $50 \AA$ $\mathrm{Cd}_{0.25} \mathrm{Zn}_{0.75}$ Se well and $\mathrm{ZnS}_{0.06} \mathrm{Se}_{0.94}$ barrier.

In summary, we have presented DLTS measurements on $n$-type $\mathrm{CdZnSSe} / \mathrm{ZnSSe}$ single quantum wells for conduction band offset determination. This system is identical to the quantum well/guiding layer heterostructure used in toady's most common blue-green II-VI separate confinement heterostructure (SCH) laser diodes (operating at $515 \mathrm{~nm}$ ). Our results indicate that the conduction band offset makes up about $75 \%$ of the band-gap difference between the barrier and quantum well. Work is being carried out to apply this characterization technique to $p$-type systems for valence band offset determination.

The authors would like to acknowledge the technical assistance of R. Breshnahan and K. Roberts and valuable discussions with colleagues at North American Philips Labora- tory. This work at the University of Michigan and $3 \mathrm{M}$ Company is supported in part by the Defense Advanced Research Projects Agency, Office of Naval Research, and the Army Research Laboratory.

${ }^{1}$ M. A. Haase, J. Qiu, J. M. DePuydt, and H. Cheng, Appl. Phys. Lett. 59, 1272 (1991).

${ }^{2}$ K. K. Law, P. F. Baude, T. J. Miller, M. A. Haase, G. M. Haugen, and K. Smekalin, Electron. Lett. (to be published).

${ }^{3}$ J. M. Gaines, R. R. Drenten, K. W. Haberern, T. Marshall, P. Mensz, and J. Petruzello, Appl. Phys. Lett. 62, 2462 (1993).

${ }^{4}$ M. A. Haase, P. F. Baude, M. S. Hagedorn, Q. Qiu, J. M. DePuydt, H. Cheng, S. Guha, G. E. Hofler, and B. J. Wu, Appl. Phys. Lett. 63, 2115 (1993).

${ }^{5}$ J. M. DePuydt, M. S. Haase, S. Guha, J. Qiu, H. Cheng, B. J. Wu, G. E. Hofler, G. Meis-Haugen, M. S. Hagedorn, and P. F. Baude, J. Cryst. Growth 138, 667 (1994).

${ }^{6}$ J. Tersoff, Phys. Rev. Lett. 56, 2755 (1986).

${ }^{7}$ M. Ukita, F. Hiei, K. Nakano, and A. Ishibashi, Appl. Phys. Lett. 66, 209 (1995).

${ }^{8}$ K. Shahzad, D. J. Olego, and C. G. Van de Walle, Phys. Rev. B 38, 1417 (1988).

${ }^{9}$ W. J. Walecki, A. V. Nurmikko, N. Samarth, H. Luo, J. K. Furdyna, and N. Otsuka, Appl. Phys. Lett. 57, 466 (1990).

${ }^{10}$ C. Trager-Cowan, P. J. Parbrook, B. Henderson, B. Henderson, and K. P. O’Donnell, Semicond. Sci. Technol. 7, 536 (1992).

${ }^{11}$ Y. Wu, K. Ichino, Y. Kawakami, S. Fijita, and S. Fujita, Jpn. J. Appl. Phys. 31, 1737 (1992).

${ }^{12}$ K. Shahzad, J. Petruzzello, J. M. Gaines, and C. Ponzoni, Appl. Phys. Lett. 67, 659 (1995).

${ }^{13}$ N. Debbar, D. Biswas, and P. Bhattacharya, Phys. Rev. B 40, 1058 (1989).

${ }^{14}$ D. Biswas, N. Debbar, P. Bhattacharya, M. Razeghi, M. Defour, and F. Omnes, Appl. Phys. Lett. 56, 833 (1990).

${ }^{15}$ D. V. Lang, in Heterojunction Band Discontinuities: Physics and Device Applications, edited by F. Capasso and G. Margaritondo (Elsevier, Amsterdam, 1987).

${ }^{16}$ B. J. Wu, J. M. DePuydt, G. M. Haugen, G. E. Hofler, M. A. Haase, H. Cheng, S. Guha, and J. Qiu, Appl. Phys. Lett. 66, 3462 (1995).

${ }^{17}$ J. Qiu, H. Cheng, J. M. DePuydt, and M. A. Haase, J. Cryst. Growth 127, 279 (1993).

${ }^{18}$ H. G. Grimmeiss, E. Meijer, R. Mach, and G. O. Muller, J. Appl. Phys. 56, 2678 (1984).

${ }^{19}$ K. Yoneda, Y. Hishida, and H. Ishii, Appl. Phys. Lett. 47, 702 (1985).

${ }^{20}$ S. Venkatesan, R. F. Pierret, J. Qiu, M. Kobayashi, R. L. Gunshor, and L. A. Kolodziejski, J. Appl. Phys. 66, 3656 (1989).

${ }^{21}$ B. Hu, G. Karczewski, H. Luo, N. Samarth, and J. K. Furdyna, J. Appl. Phys. 63, 358 (1993).

${ }^{22}$ G. Karczewski, B. Hu, A. Yin, H. Luo, N. Samarath, and J. K. Furdyna, J. Appl. Phys. 75, 7382 (1994).

${ }^{23}$ B. Hu, G. KarczewskiH. Luo, U. Bindley, and J. K. Furdyna, J. Appl. Phys. 77, 2026 (1995). 